tiguous to $P$, but increasing from one interval to another, and a sequence $\left\{m_{k}\right\}$ such that $\int_{0}^{2 \pi} \sin ^{2} m_{k} x d F<1 / k^{4}$. Hence

$$
\sum_{1}^{\infty} k^{2} \int_{0}^{2 \pi} \sin ^{2} m_{k} x d F<\infty .
$$

Hence "almost everywhere" in $P$, that is to say, in a subset $P_{1}$ of $P$ such that the variation of $F$ over $P-P_{1}$ is zero, $\sum_{1}^{\infty} k^{2} \sin ^{2} m_{k} x<\infty$. But

$$
\sum_{1}^{k}\left|\sin m_{k} x\right|<\left(\sum_{1}^{k} \frac{1}{k^{2}}\right)^{1 / 2}\left(\sum_{1}^{k} k^{2} \sin ^{2} m_{k} x\right)^{1 / 2} ;
$$

hence $\sum\left|\sin m_{k} x\right|$ converges in $P_{1}$, and $P$ is "almost everywhere" of the type $R$ (and also, almost everywhere, a set of absolute convergence).

Montreal, Canada

\title{
UNITARY SPACES WITH CORRESPONDING GEODESICS ${ }^{1}$
}

\section{N. COBURN}

1. Introduction. This paper is divided into three parts. In the first section, the notation and fundamental concepts of hermitian geometry are reviewed. The second section develops the equations of geodesic curves $X_{1}$ which depend on a real parameter $(t)$ and which are imbedded in a unitary space of $n$-dimensions $K_{n}$. Our principal result is: The equations of such geodesics differ from the equations of geodesics in Riemannian space in that the former contain the torsion affinor. In the third section, we classify the connections of two unitary spaces $K_{n},{ }^{\prime} K_{n}$ whose geodesics correspond. First, we find the necessary and sufficient conditions that two unitary spaces $K_{n},{ }^{\prime} K_{n}$ both with symmetric connection shall have their geodesics in correspondence. This last problem is solved in exactly the same manner as the similar problem in Riemannian space. ${ }^{2}$ Secondly, we prove that if $K_{n}$ has torsion and ' $K_{n}$ has no torsion (symmetric connection), then their geodesics do not correspond. The problem of determining all connections of unitary spaces $K_{n},{ }^{\prime} K_{n}$ both with torsion whose geodesics correspond is left open.

1 Presented to the Society, January 1, 1941.

${ }^{2}$ L. P. Eisenhart, Riemannian Geometry, Princeton University Press, 1926, p. 131. 
2. Notation. ${ }^{3}$ Consider a real space of $2 n$-dimensions $X_{2 n}$ whose coordinates are given by the real variables

$$
x^{\lambda}, y^{\lambda}, \quad \lambda, \mu=1 \cdots n .
$$

Into this $X_{2 n}$, we introduce the complex coordinates given by

$$
\begin{aligned}
\xi^{\lambda} & =x^{\lambda}+i y^{\lambda}, & \\
\xi^{\lambda *} & =x^{\lambda}-i y^{\lambda}, & i=(-1)^{1 / 2} .
\end{aligned}
$$

Since the Jacobian of this transformation $(-2 i)$ does not vanish over $X_{2 n}$, then $\xi^{\lambda}, \xi^{\lambda^{*}}$ constitute a set of $2 n$ independent variables which map the $X_{2 n}$. In view of the fact that $\xi^{\lambda^{*}}$ are complex conjugates to $\xi^{\lambda}$, we determine the points of $X_{2 n}$ by merely assigning complex numbers to $\xi^{\lambda}$. We say that the $\xi^{\lambda}$ determine "points" which build a complex space of $n$-dimensions $X_{n}$ (the above real topological $X_{2 n}$ ). Let us denote partial derivatives by

$$
\partial_{\mu}=\partial / \partial \xi^{\mu}, \quad \partial_{\mu^{*}}=\partial / \partial \xi^{\mu^{*}},
$$

and let $\phi\left(\xi^{\lambda}, \xi^{\lambda^{*}}\right)$ be an analytic function of the variables $\xi^{\lambda}, \xi^{\lambda^{*}}$. Then, by the composite function theorem, we obtain

$$
\begin{aligned}
& \partial \phi / \partial x^{\mu}=\partial_{\mu} \phi+\partial_{\mu^{*}} \phi, \\
& \partial \phi / \partial y^{\mu}=i \partial_{\mu} \phi-i \partial_{\mu^{*}} \phi .
\end{aligned}
$$

Solving for $\partial_{\mu} \phi, \partial_{\mu^{*}} \phi$, we find

$$
\begin{aligned}
\partial_{\mu} \phi & =1 / 2\left(\partial \phi / \partial x^{\mu}-i \partial \phi / \partial y^{\mu}\right), \\
\partial_{\mu^{*}} \phi & =1 / 2\left(\partial \phi / \partial x^{\mu}+i \partial \phi / \partial y^{\mu}\right) .
\end{aligned}
$$

If the function $\psi\left(\xi^{\lambda}\right)$,

$$
\psi\left(\xi^{\mu}\right)=u\left(x^{\lambda}, y^{\lambda}\right)+i v\left(x^{\lambda}, y^{\lambda}\right),
$$

is analytic in the sense of Cauchy-Riemann, then

$$
\begin{aligned}
& \partial u / \partial x^{\lambda}-\partial v / \partial y^{\lambda}=0, \\
& \partial v / \partial x^{\lambda}+\partial u / \partial y^{\lambda}=0 .
\end{aligned}
$$

By expanding the right-hand side of (2.7), we find that (2.9) is equivalent to

$$
\partial_{\lambda} \psi=0 .
$$

From our point of view, this equation follows directly from the fact

${ }^{3}$ J. A. Schouten and D. J. Struik, Einfuhrung in die Neueren Methoden der Differentialgeometrie, P. Noordhoff, Groningen, Batavia, vol. 2, 1938, p. 225. 
that $\xi^{\lambda}, \xi^{\lambda^{*}}$ are independent variables. Hence equation (2.9) merely serves to interpret the equation (2.10) from the point of view of complex variable. If $\psi^{*}$ is the complex conjugate function to $\psi$,

$$
\psi^{*}=u-i v,
$$

then it is easily shown that (2.9) is equivalent to the so-called conjugate equation

$$
\partial_{\lambda} \psi^{*}=0
$$

We seek to generalize the idea involved in (2.12). Corresponding to any function $\phi\left(\xi^{\lambda}, \xi^{\lambda^{*}}\right)$, let $\phi^{*}\left(\xi^{\lambda^{*}}, \xi^{\lambda}\right)$ denote the function obtained by replacing $i$ by $-i$. From (2.2), we see that the variables $\xi^{\lambda}$ are then replaced by $\xi^{\lambda^{*}}$ and conversely. We call this function $\phi^{*}$, the conjugate of $\phi$. In the future, we shall indicate the validity of the conjugate by the abbreviation "conj." It is to be noted on the formal side, that in passing to the conjugate, all indices will be starred. The star of a starred quantity removes the original star.

Consider the allowable analytic coordinate transformations with nonvanishing Jacobian

$$
\xi^{\lambda}=\xi^{\lambda}\left(\xi^{\lambda^{\prime}}\right), \quad \xi^{\lambda^{\prime}}=x^{\lambda^{\prime}}+i y^{\lambda^{\prime}} .
$$

The corresponding conjugate equations are

$$
\xi^{\lambda^{*}}=\xi^{\lambda^{*}}\left(\xi^{\lambda^{* \prime}}\right), \quad \xi^{\lambda^{* \prime}}=x^{\lambda^{\prime}}-i y^{\lambda^{\prime}} .
$$

We now introduce the unit affinor whose intermediary components are

$$
A_{\mu^{\prime}}^{\lambda}=\partial \xi^{\lambda} / \partial \xi^{\mu^{\prime}}, \quad A_{\mu^{* \prime}}^{\lambda^{*}}=\partial \xi^{\lambda^{*}} / \partial \xi^{\mu^{* \prime}} \text {. }
$$

A vector $v^{\lambda}\left(\xi^{\lambda}, \xi^{\lambda^{*}}\right)$ is said to be of the first type if

$$
v^{\lambda^{\prime}}=A_{\lambda}^{\lambda^{\prime}} v^{\lambda} \text {. }
$$

Associated with each such vector is its conjugate, or vector of the second type $v^{\lambda *}$, with transformation law

$$
v^{\lambda^{* \prime}}=A_{\lambda^{*}}^{\lambda^{* \prime} v^{\lambda^{*}}} \text {. }
$$

The theory can be extended to affinors of any mixed valence.

Let us introduce a connection in $X_{n}$, by means of the $n^{3}$ quantities $\Gamma_{\mu \alpha}^{\lambda}$ which are functions of position. Then, we define the covariant derivative of a contravariant vector by

$$
\delta v^{\lambda}=d v^{\lambda}+\Gamma_{\mu \alpha}^{\lambda} v^{\alpha} d \xi^{\mu} \text {, conj. }
$$


The $\Gamma_{\mu \alpha}^{\lambda}$ transform in a well known manner ${ }^{4}$ under (2.13). We now write for a covariant vector

$$
\delta w_{\lambda}=d w_{\lambda}-\Gamma_{\mu \lambda}^{\alpha} w_{\alpha} d \xi^{\mu}, \text { conj. }
$$

By expanding the ordinary differential of a vector, we obtain

$$
d v^{\lambda}=d \xi^{\mu} \partial_{\mu} v^{\lambda}+d \xi^{\mu^{*}} \partial_{\mu^{*}} v^{\lambda}, \text { conj. }
$$

If we define the covariant derivative of $v^{\lambda}, w^{\lambda}$ by means of

$$
\begin{aligned}
\nabla_{\mu} v^{\lambda}=\partial_{\mu} v^{\lambda}+\Gamma_{\mu \alpha}^{\lambda} v^{\alpha}, & \nabla_{\mu} w_{\lambda}=\partial_{\mu} w_{\lambda}-\Gamma_{\mu \lambda}^{\alpha} w_{\alpha}, \\
\nabla_{\mu \nu}{ }^{\lambda}=\partial_{\mu \nu}{ }^{\lambda}, & \nabla_{\mu} w_{\lambda}=\partial_{\mu *} w_{\lambda}, \text { conj. },
\end{aligned}
$$

then

$$
\begin{aligned}
\delta v^{\lambda} & =d \xi^{\mu} \nabla_{\mu} v^{\lambda}+d \xi^{\mu^{*}} \nabla_{\mu^{*} v^{\lambda}}, \text { conj., } \\
\delta w_{\lambda} & =d \xi^{\mu} \nabla_{\mu} w_{\lambda}+d \xi^{\mu^{*}} \nabla_{\mu^{*}} w_{\lambda}, \text { conj., }
\end{aligned}
$$

An hermitian $X_{n}$, with covariant derivative defined by (2.21), (2.22) is denoted by $K_{n}$.

We now introduce an hermitian tensor with hermitian symmetry

$$
a_{\lambda \mu^{*}}=\left(a_{\lambda \mu^{*}}\right)^{* \prime}=a_{\mu^{*} \lambda},
$$

the (') indicating the transpose matrix. If we condition $a_{\lambda \mu^{*}}$ by requiring that

$$
\delta a_{\lambda \mu^{*}}=0=\left(\partial_{\nu} a_{\lambda \mu^{*}}-\Gamma_{\nu \lambda}^{\rho} a_{\rho \mu^{*}}\right) d \xi^{\nu}+\left(\partial_{\nu^{*}} a_{\lambda \mu^{*}}-\Gamma_{\nu^{*} \mu^{*}}^{\rho^{*}} a_{\lambda \rho^{*}}\right) d \xi^{\xi^{*}},
$$

then the space $K_{n}$ is said to be a unitary $K_{n}$. For such a space, from (2.26), we can prove ${ }^{5}$

$$
\begin{aligned}
\nabla_{\nu} a_{\lambda \mu^{*}} & =\partial_{\nu} a_{\lambda \mu^{*}}-\Gamma_{\nu \lambda}^{\rho} a_{\rho \mu^{*}}=0, \text { conj., } \\
\nabla_{\nu^{*}} a_{\lambda \mu^{*}} & =\partial_{\nu^{*}} a_{\lambda \mu^{*}}-\Gamma_{\nu^{*} \mu^{*}}^{\rho^{*}} a_{\lambda \rho^{*}}=0, \text { conj. }
\end{aligned}
$$

The $a_{\lambda \mu^{*}}$ is now a fundamental tensor and can be used to raise and lower indices through the $\nabla$ operator. If we define the contravariant fundamental tensor $a^{\sigma^{*} \kappa}$ by

$$
a^{\sigma^{*} \kappa} a_{\lambda \sigma^{*}}=A_{\lambda}^{\kappa}, \text { conj., }
$$

then (2.27), (2.28) may be solved for the connection

$$
\begin{aligned}
\Gamma_{\mu \lambda}^{\kappa} & =\left(\partial_{\mu} a_{\lambda \mu^{*}}\right) a^{\sigma^{*} \kappa}=\left(\partial_{\mu} a_{\sigma^{*} \lambda}\right) a^{\alpha^{*}}, \\
\Gamma_{\mu^{*} \lambda^{*}}^{\kappa^{*}} & =\left(\partial_{\mu^{*}} a_{\sigma \lambda^{*}}\right) a^{\kappa^{*} \sigma}=\left(\partial_{\mu^{*}} a_{\lambda^{*} \sigma}\right) a^{\sigma \kappa^{*}} .
\end{aligned}
$$

${ }^{4}$ J. A. Schouten and D. J. Struik, loc. cit., p. 227.

5 J. A. Schouten and D. J. Struik, loc. cit., p. 234. 
Finally, we introduce the torsion affinor

$$
{S_{\mu \lambda}}_{\mu \alpha}^{*}=1 / 2\left(\Gamma_{\mu \lambda}^{\kappa}-\Gamma_{\lambda \mu}^{\kappa}\right)=\Gamma_{[\mu \lambda]}^{\kappa} \text {, conj. }
$$

The sign [ ] means that the antisymmetric product of the enclosed indices is to be formed; the sign ( ) means that the symmetric product of the enclosed indices is to be formed.

3. Equations of geodesics in $K_{n}$. Consider the curve $X_{1}$ defined in $K_{n}$ by means of the equations

$$
\xi^{\lambda}=\xi^{\lambda}(t), \quad \xi^{\lambda^{*}}=\xi^{\lambda^{*}}(t)
$$

where $(t)$ is a real parameter and $\xi^{\lambda}, \xi^{\lambda^{*}}$ are conjugate functions. Evidently, such equations determine a curve $X_{1}$ in the real topological $X_{2 n}$. We define the element of arc length along this $X_{1}$ by

$$
d s=\left(a_{\lambda \mu^{*}} d_{t} \xi^{\lambda} d_{t} \xi^{\mu^{*}}\right)^{1 / 2} d t .
$$

By substituting (3.1) into (3.2) and integrating, we obtain ( $s$ ) the arc length parameter. It is to be noted that this parameter exists only because of the fact that $(t)$ is real. By use of (2.25) and the conjugate of (3.2), we see that $(d s)$ and hence $(s)$ are real.

We follow the well known methods of the calculus of variations ${ }^{6}$ in finding the geodesics of $K_{n}$. Our problem is to find that $X_{1}$ for which the first variation of

$$
I=\int_{t_{0}}^{t_{1}}\left(a_{\lambda \mu^{*}} d_{t} \xi^{\lambda} d_{t} \xi^{*}\right)^{1 / 2} d t
$$

is zero. Let $\xi^{\lambda}$ represent the coordinates of a point on the geodesic $X_{1}$; let ' $\xi \lambda$ represent the coordinates of a point on the varied ' $X_{1}$; let $\epsilon$ be an infinitesimal; and let $\omega^{\lambda}$ be an arbitrary function of $\xi^{\lambda}, \xi^{\lambda^{*}}$ which vanishes at $t=t_{0}, t_{1}$. The equations of variation can be written as

$$
' \xi^{\lambda}=\xi^{\lambda}+\epsilon \omega^{\lambda}, \text { conj. }
$$

For clarity, we write

$$
\phi\left(\xi^{\lambda}, \xi^{\lambda^{*}}, \dot{\xi}^{\lambda}, \dot{\xi}^{\lambda *}\right)=\left(a_{\lambda \mu^{*}} d_{t} \xi^{\xi^{\lambda}} d_{t} \xi^{\xi^{*}}\right)^{1 / 2},
$$

where

$$
\dot{\xi}^{\lambda}=d_{t} \xi^{\lambda}, \quad \dot{\xi}^{\lambda *}=d_{t} \xi^{\lambda *} .
$$

Then by use of Taylor's series and integration by parts, we obtain for the first variation of $(3.3)$

${ }^{6}$ L. P. Eisenhart, loc. cit., p. 49. 
(3.7) $0=\int_{t_{0}}^{t_{1}}\left[\frac{\partial \phi}{\partial \xi^{\lambda}}-\frac{d}{d t}\left(\frac{\partial \phi}{\partial \dot{\xi}^{\lambda}}\right)\right] \omega^{\lambda} d t+\left[\frac{\partial \phi}{\partial \xi^{\lambda^{*}}}-\frac{d}{d t}\left(\frac{\partial \phi}{\partial \dot{\xi}^{\lambda^{*}}}\right)\right] \omega^{\lambda^{*}} d t$.

First, let us assume that the vector $\left(\omega^{\lambda}, \omega^{\lambda^{*}}\right)$ has the components $(a, b, \cdots, a, b, \cdots)$ where $a, b, \cdots$ are arbitrary real functions of $\xi^{\lambda}, \xi^{\lambda^{*}}$. Secondly, let us assume that $\left(\omega^{\lambda}, \omega^{\lambda^{*}}\right)$ has the components $(i a, i b, \cdots,-i a,-i b, \cdots)$. Then, by substituting into (3.7), we find that this relation decomposes in to the two equations

$$
\begin{aligned}
& 0=\int_{t_{0}}^{t_{1}}\left[\frac{\partial \phi}{\partial \xi^{\lambda}}-\frac{d}{d t}\left(\frac{\partial \phi}{\partial \xi^{\lambda}}\right)\right] \omega^{\lambda} d t, \\
& 0=\int_{t_{0}}^{t_{1}}\left[\frac{\partial \phi}{\partial \xi^{\lambda^{*}}}-\frac{d}{d t}\left(\frac{\partial \phi}{\partial \dot{\xi}^{\lambda^{*}}}\right)\right] \omega^{\lambda *} d t .
\end{aligned}
$$

By the ordinary argument, we obtain the Euler equations

$$
\begin{aligned}
& \frac{\partial \phi}{\partial \xi^{\lambda}}-\frac{d}{d t}\left(\frac{\partial \phi}{\partial \dot{\xi}^{\lambda}}\right)=0, \\
& \frac{\partial \phi}{\partial \xi^{\lambda *}}-\frac{d}{d t}\left(\frac{\partial \phi}{\partial \dot{\xi}^{\lambda *}}\right)=0 .
\end{aligned}
$$

We follow Eisenhart in listing the steps of our computation. From (3.5), we have

$$
\begin{gathered}
\frac{\partial \phi}{\partial \xi^{\lambda}}=\frac{1}{2} \frac{a_{\lambda \mu^{*}} d_{t} \xi^{\mu^{*}}}{\left(a_{\lambda \mu^{*}} d_{t} \xi^{\lambda} d_{t} \xi^{\mu^{*}}\right)^{1 / 2}}=\frac{1}{2} \frac{a_{\lambda \mu^{*}} d_{t} \xi^{\mu^{*}}}{(d s / d t)} \\
\frac{\partial \phi}{\partial \xi^{\lambda}}=\frac{1}{2} \frac{\left(\partial_{\lambda} a_{\nu \mu^{*}}\right) d_{t} \xi^{\nu} d_{t} \xi^{\mu^{*}}}{(d s / d t)}, \\
\frac{d}{d t}\left(\frac{\partial \phi}{\partial \dot{\xi}^{\lambda}}\right) \\
=\frac{1}{2} \frac{\frac{d s}{d t}\left[a_{\lambda \mu^{*}} d_{t}^{2} \xi^{\mu^{*}}+\partial_{\nu} a_{\lambda \mu^{*}} d_{t} \xi^{\nu} d_{t} \xi^{\mu^{*}}+\partial_{\nu^{*}} a_{\lambda \mu^{*}} d_{t} \xi^{\nu^{*}} d_{t} \xi^{\mu^{*}}\right]-\frac{d^{2} s}{d t^{2}}\left(a_{\lambda \mu^{*}} d_{t} \xi^{\mu^{*}}\right)}{(d s / d t)^{2}} .
\end{gathered}
$$

Substituting these relations into (3.5), we obtain

$$
a_{\lambda \mu^{*}} d_{t}^{2} \xi^{\mu^{*}}+\partial_{\nu} a_{\lambda \mu^{*}} d_{t} \xi^{\nu} d_{t} \xi^{\mu^{*}}+\partial_{\nu^{*}} a_{\lambda \mu^{*}} d_{t} \xi^{\nu^{*}} d_{t} \xi^{\mu^{*}}-\partial_{\lambda} a_{\nu \mu^{*}} d_{t} \xi^{\nu} d_{t} \xi^{\mu^{*}}
$$

$$
-\frac{a_{\lambda \mu^{*}} d_{t} \xi^{\mu^{*}} \frac{d^{2} s}{d t^{2}}}{(d s / d t)}=0
$$


From (2.30) and (2.31), we find

$$
\begin{aligned}
\partial_{\nu} a_{\lambda \mu^{*}} & =\Gamma_{\nu \lambda}^{\kappa} a_{\kappa \mu^{*}}, \\
\partial_{\nu^{*}} a_{\lambda \mu^{*}} & =\Gamma_{\nu^{*} \mu^{*}}^{\kappa^{*}} a_{\lambda \kappa^{*}} .
\end{aligned}
$$

Hence (3.15) becomes

$$
\begin{gathered}
a_{\lambda \mu^{*}} d_{t}^{2} \xi^{\mu^{*}}+a_{\kappa \mu^{*} \Gamma_{\nu \lambda}}{ }_{\nu} d_{t} \xi^{\nu} d_{t} \xi^{\mu^{*}}+\Gamma_{\nu^{*} \mu^{*}}^{\kappa^{*}} a_{\lambda \kappa^{*}} d_{t} \xi^{\nu^{*}} d_{t} \xi^{\mu^{*}}-\Gamma_{\lambda \nu}^{\kappa} a_{\kappa \mu^{*}} d_{t} \xi^{\nu} d_{t} \xi^{\mu^{*}} \\
-\frac{a_{\lambda \mu^{*}} d_{t} \xi^{\lambda^{*}} \frac{d^{2} s}{d t^{2}}}{(d s / d t)}=0 .
\end{gathered}
$$

Transvecting (3.18) with $a^{\alpha^{* \lambda}}$, we obtain after combining terms two and four

$$
d_{t}^{2} \xi^{\alpha^{*}}+\Gamma_{\nu^{*} \mu^{*}}^{\alpha^{*}} d_{t} \xi^{\nu^{*}} d_{t} \xi^{\mu^{*}}+2 a_{\kappa \mu^{*}} a^{\alpha^{*} \lambda} S_{\nu \lambda}^{\cdots^{\kappa}} d_{t} \xi^{\nu} d_{t} \xi^{\mu^{*}}-d_{t} \xi^{\alpha^{*}}\left(\frac{d^{2} s}{d t^{2}} / \frac{d s}{d t}\right)=0 .
$$

From the equation (3.11), we obtain the conjugate of (3.19). Hence, we have this theorem:

TheOREM 1. The geodesic $X_{1}$ in unitary $K_{n}$ which are functions of a real parameter $(t)$ is given by the solutions of the second order differential equation (3.19) and its conjugate.

Evidently, the equations of the geodesic $X_{1}$ in unitary $K_{n}$ differ from the equations of the geodesic $V_{1}$ in $V_{n}$ in the additional term containing the torsion affinor. It follows that if the unitary space $K_{n}$ has no torsion, then the geodesic $X_{1}$ of $K_{n}$ satisfies the same type of differential equation as the geodesic $V_{1}$ in $V_{n}$ and conversely.

4. Unitary spaces with corresponding geodesics. By multiplying the conjugate of (3.19) by $d_{t} \xi^{\beta}$, forming a similar equation with the indices $\alpha, \beta$ interchanged and then subtracting the two resulting equations, we obtain ${ }^{7}$ for the geodesic $X_{1}$ in $K_{n}$

$$
\begin{aligned}
\left(d_{t} \xi^{\beta} d_{t}^{2} \xi^{\alpha}-d_{t} \xi^{\alpha} d_{t}^{2} \xi^{\beta}\right)+\left(\Gamma_{\nu \mu}^{\alpha} d_{t} \xi^{\beta}-\Gamma_{\nu \mu}^{\beta} d_{t} \xi^{\alpha}\right) d_{t} \xi^{\nu} d_{t} \xi^{\mu} & \\
& +2 a_{\mu \kappa^{*} \cdot S_{\nu^{*} \lambda^{*}}}\left(a^{\alpha^{*} \alpha} d_{t} \xi^{\beta}-a^{\lambda^{*} \beta} d_{t} \xi^{\alpha}\right) d_{t} \xi^{\nu^{*}} d_{t} \xi^{\mu}=0, \text { conj. }
\end{aligned}
$$

Consider another $n$-dimensional unitary space ' $K_{n}$ which is also mapped by the variables $\xi^{\lambda}$ such that the point $P\left(\xi^{\lambda}\right)$ of $K_{n}$ corresponds to the point ' $P\left(\xi^{\lambda}\right)$ of ' $K_{n}$. Furthermore, let the fundamental tensor and connection of this space be denoted by

$$
{ }^{\prime} a_{\lambda \mu^{*}}, \quad{ }^{\prime} \Gamma_{\nu \mu}^{\kappa}
$$

' L. P. Eisenhart, loc. cit., p. 131. 
If the geodesics of ' $K_{n}$ correspond to those of $K_{n}$, then equation (4.1) with $a_{\lambda \mu^{*}}, \Gamma_{\lambda \mu}^{\alpha}, S_{\nu \mu}{ }^{{ }^{k}}$ replaced by ' $a_{\lambda \mu^{*}},{ }^{\prime} \Gamma_{\lambda \mu}^{\alpha},{ }^{\alpha} S_{\nu \mu}{ }^{{ }^{k}}$ is satisfied by the same functions $\xi^{\alpha}(t)$ which satisfy (4.1), that is,

$$
\begin{aligned}
& \left(d_{t} \xi^{\beta} d_{t}^{2} \xi^{\alpha}-d_{t} \xi^{\alpha} d_{t}^{2} \xi^{\beta}\right)+\left({ }^{\prime} \Gamma_{\nu \mu}^{\alpha} d_{t} \xi^{\beta}-{ }^{\prime} \Gamma_{\nu \mu}^{\beta} d_{t} \xi^{\alpha}\right) d_{t} \xi^{\nu} d_{t} \xi^{\mu} \\
& +2{ }^{\prime} a_{\mu \kappa^{*}}{ }^{\prime} S_{\nu^{*} \lambda^{*}}^{\cdot \kappa^{*}}\left({ }^{\prime} a^{\lambda^{*} \alpha} d_{t} \xi^{\beta}-{ }^{\prime \lambda^{* \beta}} d_{t} \xi^{\alpha}\right) d_{t} \xi^{\xi^{*}} d_{t} \xi^{\mu}=0 \text {, conj. }
\end{aligned}
$$

Let us first assume that $K_{n}$ and ' $K_{n}$ both possess symmetric connections.

THEOREM 2. The necessary and sufficient condition that two unitary spaces $K_{n},{ }^{\prime} K_{n}$ both with symmetric connections have geodesics in correspondence is that a vector $p_{\nu}$ exist such that

$$
{ }^{\prime} \Gamma_{\nu \mu}^{\alpha}=\Gamma_{\nu \mu}^{\alpha}+2 p_{(\nu} A_{\mu)}^{\alpha}, \text { conj. }
$$

We can write the equation

$$
{ }^{\prime} \Gamma_{\nu \mu}^{\alpha}=\Gamma_{\nu \mu}^{\alpha}+A_{\nu \mu}^{\cdots \alpha}, \text { conj. }
$$

where $A_{\nu \mu}{ }^{\alpha}$ is an affinor to be determined. Subtracting (4.1) from (4.3) (note: the torsion affinors are zero in this case), we obtain

$$
\left(\stackrel{A_{\nu \mu}^{* \alpha}}{A_{t} \xi^{\beta}}-\stackrel{A_{\nu \mu}^{* \beta}}{A_{t} \xi^{\alpha}}\right) d_{t} \xi^{\nu} d_{t} \xi^{\mu}=0 \text {, conj. }
$$

By use of the unit affinor $A_{\gamma}^{\alpha}$, we may rewrite (4.5) in the simpler form

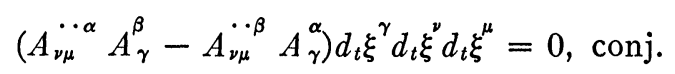

Since (4.6) is to be satisfied by arbitrary values of $d_{t} \xi^{\alpha}$ at any point $P\left(\xi^{\lambda}\right)$ and ${ }^{\prime} P\left(\xi^{\lambda}\right)$, we find

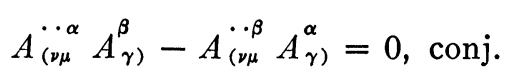

Expanding (4.7), we obtain

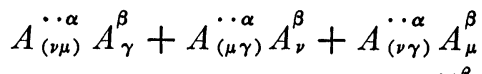

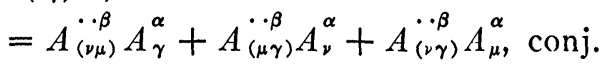

Let us write

$$
\stackrel{A_{\nu \alpha}^{* \alpha}}{A_{\nu \alpha}^{* \alpha}} \stackrel{A_{\alpha \nu}^{* \alpha}}{A^{2}}(n+1) p_{\nu}, \text { conj., }
$$

and contract on the indices $\mu, \alpha$ in (4.8). Then we obtain

$$
\stackrel{A_{\nu \gamma}^{* \beta}}{\stackrel{* \beta}{*}} 2 p_{(\nu} A_{\gamma)}^{\beta} \text {, conj. }
$$


Conversely, if (4.10) is satisfied, then (4.6) is identically satisfied. Hence our theorem is proved.

Let us now assume that $K_{n}$ has torsion and ' $K_{n}$ has no torsion (symmetric connection).

TheOREM 3. Two unitary spaces, $K_{n}$ with torsion, ' $K_{n}$ without torsion, cannot have their geodesics in correspondence.

Let us assume the contrary, namely, that the geodesics of these two spaces correspond. Then again, we write the equation (4.4) and again, we subtract (4.1) from (4.3). In this case, instead of equation (4.6), we obtain

$$
\begin{aligned}
& \left(A_{\nu \mu}^{\cdots \alpha} A_{\gamma}^{\beta}-\stackrel{A_{\nu \mu}^{* \beta}}{A^{*}} A_{\gamma}^{\alpha}\right) d_{t} \xi^{\gamma} d_{t} \xi^{\nu} d_{t} \xi^{\mu}
\end{aligned}
$$

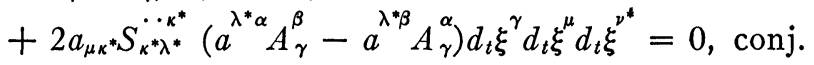

Equation (4.11) is to be satisfied by arbitrary values of the $d_{t} \xi^{\alpha}$. Let us first assume that the vector $\left(d_{t} \xi^{\alpha}, d_{t} \xi^{\alpha^{*}}\right)$ has the arbitrary real components $(a, b, c, \cdots a, b, c, \cdots)$ and secondly, let us assume that this vector has the arbitrary pure imaginary components $(i a, i b, i c, \cdots-i a,-i b, \cdots)$. By adding the resulting equations, we find that (4.11) decomposes into the two equations

$$
\begin{gathered}
\left(A_{\nu \mu}^{\cdots \alpha} A_{\gamma}^{\beta}-A_{\nu \mu}^{\cdots \beta} A_{\gamma}^{\alpha}\right) d_{t} \xi^{\gamma} d_{t} \xi^{\nu} d_{t} \xi^{\mu}=0 \text {, conj., } \\
a_{\mu \kappa^{*}} S_{\nu^{*} \lambda^{*}}^{\cdots \kappa^{*}}\left(a^{\lambda^{*} \alpha} A_{\gamma}^{\beta}-a^{\lambda^{*} \beta} A_{\gamma}^{\alpha}\right) d_{t} \xi^{\gamma} d_{t} \xi^{\mu} d_{t} \xi^{\nu^{*}}=0 \text {, conj. }
\end{gathered}
$$

The equation (4.12) can be analyzed in an exactly analogous manner to that used in the previous theorem excepting that (4.9) must be replaced by

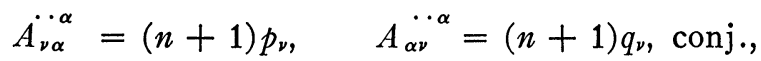

since $A_{\nu \mu}^{\stackrel{\alpha}{\alpha}}$ is no longer symmetric in $\nu, \mu$. Instead of (4.10), we find

$$
\underset{(\nu \gamma)}{\stackrel{* \beta}{A}}=2 r_{(\nu} A_{\gamma)}^{\beta}, \quad 2 r_{\nu}=p_{\nu}+q_{\nu} \text { conj. }
$$

In order to analyze (4.13), we write this equation in the form

$$
A_{\mu \gamma \nu^{*}}^{\alpha \beta} d_{t} \xi^{\mu} d_{t} \xi^{\gamma} d_{t} \xi^{\nu^{*}}=0 \text {, conj., }
$$

where

$$
A_{\mu \gamma \nu^{*}}^{\alpha \beta}=a_{\mu \kappa^{*}} S_{\nu^{*} \lambda^{*}}^{\cdots \kappa}\left(a^{\lambda^{*} \alpha} A_{\gamma}^{\beta}-a^{\lambda^{*} \beta} A_{\gamma}^{\alpha}\right) \text {, conj. }
$$

As previously, let us assume first that $\left(d_{t} \xi^{\alpha}, d_{t} \xi^{\alpha^{*}}\right)$ has the arbitrary real components $(a, b, \cdots a, b, \cdots)$. Upon substituting into (4.16), we obtain a third degree homogeneous polynomial. We shall explicitly 
write those terms which contain $a, b$; the remaining terms are formulated in a similar fashion. We have

$$
\begin{aligned}
A_{111^{*}}^{\alpha \beta} a^{3} & +a^{2} b\left(A_{112^{*}}^{\alpha \beta}+A_{121^{*}}^{\alpha \beta}+A_{211^{*}}^{\alpha \beta}\right) \\
& +a b^{2}\left(A_{122^{*}}^{\alpha \beta}+A_{212^{*}}^{\alpha \beta}+A_{221^{*}}^{\alpha \beta}\right)+A_{222^{*} b^{3}}^{\alpha \beta}+\cdots=0 .
\end{aligned}
$$

Secondly, let us assume $\left(d_{t} \xi^{\alpha}, d_{t} \xi^{*}\right)$ has the arbitrary components $(a, i b, \cdots a, i b, \cdots)$. Then (4.16) becomes

$$
\begin{aligned}
A_{111^{*}}^{\alpha \beta} & a^{3}+a^{2} b i\left(-A_{112^{*}}^{\alpha \beta}+A_{121^{*}}^{\alpha \beta}+A_{211^{*}}^{\alpha \beta}\right) \\
& +a b^{2}\left(A_{122^{*}}^{\alpha \beta}+A_{212^{*}}^{\alpha \beta}-A_{221^{*}}^{\alpha \beta}\right)-i A_{222^{*} b^{3}}^{\alpha \beta}+\cdots=0 .
\end{aligned}
$$

From these equations, that is, (4.18), (4.19) we conclude

$$
A_{111^{*}}^{\alpha \beta}=A_{222^{*}}^{\alpha \beta}=A_{122^{*}}^{\alpha \beta}=A_{221^{*}}^{\alpha \beta}=0, \quad A_{(12) 1^{*}}^{\alpha \beta}=A_{(12) 2^{*}}^{\alpha \beta}=0 .
$$

We can evidently express $(4.20)$ by writing

$$
A_{(\mu \gamma) \nu^{*}}^{\alpha \beta}=0 \text {, conj. }
$$

By use of (4.17), we find upon expanding

$$
S_{\nu^{*} \lambda^{*}}^{\cdot \kappa^{*}}\left(a_{\mu \kappa^{*}} a^{\lambda^{*} \alpha} A_{\gamma}^{\beta}+a_{\gamma \kappa^{*}} a^{\lambda^{*} \alpha} A_{\mu}^{\beta}-a_{\mu \kappa^{*}} a^{\lambda^{*} \beta} A_{\gamma}^{\alpha}-a_{\gamma \kappa^{*}} a^{\lambda^{*} \beta} A_{\mu}^{\alpha}\right)=0 \text {, conj. }
$$

Placing $\alpha$ equal to $\mu$ and contracting, we find

$$
S_{\nu^{*} \lambda^{*}}^{\cdot \lambda^{*}} A_{\gamma}^{\beta}=n S_{\nu^{*} \lambda^{*}}^{\cdots \kappa^{*}} a_{\gamma^{*}} a^{\lambda^{*} \beta} \text {, conj. }
$$

Solving (4.23) for $S_{\nu^{*} \lambda^{*}}^{*}$, we find

$$
S_{\nu^{*} \mu^{*}}^{* \alpha^{*}}=S_{\nu^{*}} A_{\mu^{*}}^{\alpha^{*}}, \text { conj., }
$$

where

$$
S_{\nu^{*} \lambda^{*}}^{\cdot \cdot \lambda^{*}}=n S_{\nu^{*}}, \text { conj. }
$$

Since $S_{\nu^{*} \lambda^{*}}^{\alpha^{*}}$ is antisymmetric in $\nu^{*}, \mu^{*}$ we have

$$
S_{\left(\nu^{*}\right.} A_{\left.\mu^{*}\right)}^{\alpha^{*}}=0 \text {, conj. }
$$

Contracting on $\alpha^{*}, \mu^{*}$ we obtain the relation

$$
S_{\nu^{*}}=0, \text { conj. }
$$

Hence $S_{\nu^{*} \mu^{*}} \kappa^{*}$ vanishes. But this is contrary to our assumption. Hence our theorem is proved.

UNIVERSITY OF TEXAS 